\title{
Mesure de la texture des fromages à l'aide d'un appareil simple
}

\author{
par \\ D. LE BARS et J.L. BERGERE \\ I.N.R.A., Laboratoire de Biochimie Microbienne, C.N.R.Z. \\ 78350 Jouy-en-Josas (France)
}

\section{INTRODUCTION}

Les fromages affinés subissent des transformations profondes entre le moment où ils sont fabriqués et celui où ils sont consommables.

Parmi ces transformations, la plus importante est la dégradation des protéines qui est responsable des principales modifications de texture affectant les fromages au cours de leur affinage, ceci est particulièrement net dans les fromages à pâte molle.

Ce phénomène est gouverné par la présence et surtout par le développement et l'activité des micro-organismes présents dans les fromages, c'est-à-dire par la nature et la quantité des enzymes protéolytiques qu'ils élaborent et par l'action de ces enzymes sur les constituants du fromage.

A l'heure actuelle on commence à connaître l'équipement enzymatique protéolytique de quelques-uns de ces micro-organismes et les propriétés des enzymes qui les constituent mais on n'a pas de données sur le rôle et la part qu'ils jouent dans le processus de l'affinage des fromages. Pour les étudier on dispose de méthodes biochimiques mais il serait également nécessaire de déterminer directement par des méthodes instrumentales les modifications de texture dont ils sont responsables.

Comme pour les autres produits alimentaires [9], plusieurs types d'appareils ont été mis au point et utilisés pour l'appréciation de la texture des fromages et le plus souvent d'ailleurs celle des fromages fondus. Ce sont par exemple le "Ball Compressor " par Scott Blair et Coppen [15], des sectilomètres par Caffyn [4], Emmon et Price [6] et Fukushima et al. [7], des " écrasomètres " par Templeton et Sommer [19] et Davis [5], un consistomètre par Bohac [2], un plastomètre par Rogers et Sanders [14], des pénétromètres à aiguilles par Baron [1] et à cônes ou billes par Fukushima et al. [7] et 
Thomas et al. [20], des appareils à mesurer l'émiettement par Thomas et al. [20] ou la cohésion par Mirgorodsky [11] et surtout des appareils d'étude du fluage conçus d'après les travaux de Davis [5] sur différents produits laitiers dont les fromages. Avec ce type d'appareil, Mulder [12], puis Raadsweld et Mulder [13] ont étudié les variations de texture de l'Edam au cours de l'affinage en fonction de la température et du $\mathrm{pH}$, et Szabo a étudié celle du fromage fondu en fonction de plusieurs paramètres [18].

Des appareils qualifiés d'universels, parce qu'ils permettent d'exécuter plusieurs types de tests et ont des possibilités de réglage ont également été utilisés pour le fromage, c'est le cas pour l' «Universal Testing Machine Instron " par Shama et Sherman [16] ou le "General Food Texturometer » par Brennan et al. [3]. Si ces derniers appareils présentent beaucoup d'avantages et sont commercialisés ils ont l'inconvénient d'être coûteux.

Pour nos études sur le rôle des enzymes protéolytiques dans la maturation des fromages, nous avons donc été amenés à mettre au point un appareil simple pour apprécier la texture.

Dans ce qui suit nous décrivons cet appareil, la méthode de mesure utilisée et à titre d'exemple les résultats obtenus sur un fromage.

\section{DESCRIPTION DE L'APPAREIL}

Cet appareil dérivé du « Rhéomètre » conçu par Davis en 1937 [5], permet d'effectuer des essais de déformation provoqués par une contrainte constante (essai de retard) suivis d'une mesure de recouvrance élastique.

Le bâti de l'appareil comprend une plaque de base horizontale (a) et une plaque verticale (b) sur laquelle sont fixés les différents composants (fig. 1 A). L'échantillon E est placé entre un socle métallique (S) posé sur la plaque de base (a) et le plateau de compression qui est un disque horizontal de $30 \mathrm{~mm}$ de diamètre (1) solidaire d'une tige métallique (2) de $12 \mathrm{~cm}$ de long qui coulisse verticalement entre deux guides (3). L'ensemble de compression plateau-tige est suspendu à un fil métallique inextensible (4) qui le relie, par l'intermédiaire d'une poulie verticale (5) à la partie mobile d'un capteur de déplacement linéaire à transformateur différentiel Cimarec type C 100 (6). L'ensemble mobile constitué par le plateau, la tige, le fil et le noyau du capteur est équilibré par un contrepoids (7). Il est possible de régler horizontalement la position de la poulie et des guides afin que les tiges du capteur et du plateau de compression coulissent sans frottement. Le capteur est alimenté par une pile au mercure de $8,3 \mathrm{v}$, et sa tension de sortie est mesurée sur un enregistreur potentiométrique. La variation de cette tension est proportion- 
A

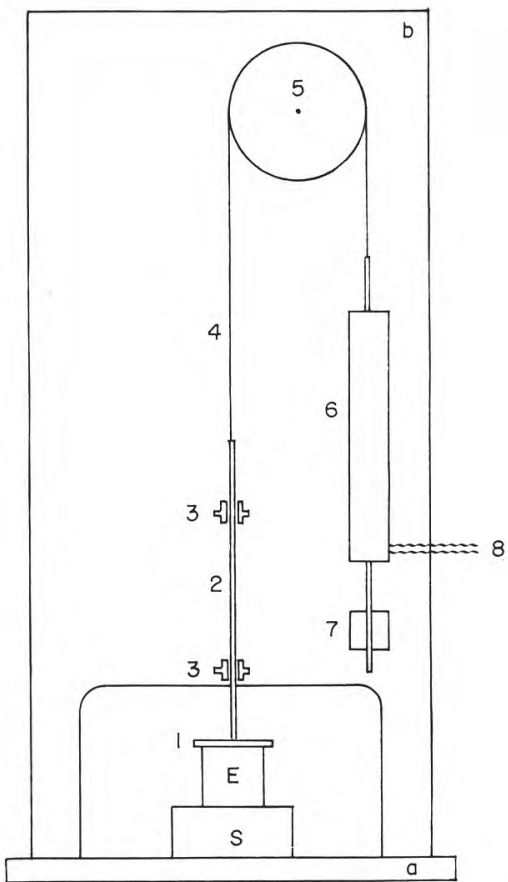

B

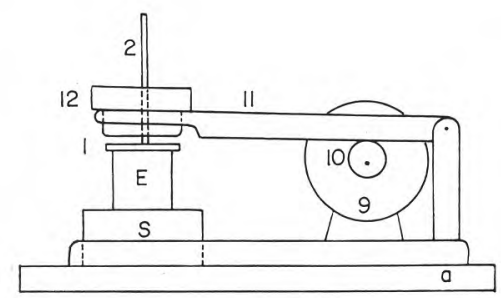

fig. 1

Schéma de l'appareil de mesure de la texture (A) et du dispositif de dépôt des poids (B).

(Les différentes parties de l'appareil, de 1 à $12, \mathrm{a}, \mathrm{b}$ et $\mathrm{s}$ sont décrites dans le texte).

nelle au déplacement de la partie mobile du capteur, la courbe enregistrée représente donc la déformation de l'échantillon en fonction du temps.

Un dispositif automatique (fig. $1 \mathrm{~B}$ ) permet de poser ou de retirer un poids circulaire fendu (12) sur le plateau de compression (1). Il comprend un micromoteur (9) sur l'axe duquel est fixée une came (10) qui fait monter ou descendre en $2 \mathrm{~s}$ une fourchette qui soulève ou repose le poids (12). Une minuterie permet de programmer la durée des cycles de compression (v. § suivant). 


\section{METHODE ET MESURE}

On découpe dans le fromage à étudier un cylindre de $24 \mathrm{~mm}$ de diamètre, haut de $24 \mathrm{~mm}$ à l'aide d'un emporte-pièces, puis d'un fil d'acier tendu. Cette éprouvette (E) est déposée dans un récipient dont l'amosphère est saturée en vapeur d'eau et l'ensemble placé dans la salle de mesure où la température est maintenue à $20^{\circ} \mathrm{C} \pm$ $0,5^{\circ} \mathrm{C}$. En $1 \mathrm{~h}$ la température de l'échantillon est équilibrée.

L'appareil est d'abord étalonné à l'aide de cales de précision de 23-24 et $25 \mathrm{~mm}$ de hauteur.

L'éprouvette de fromage est ensuite placée sous le plateau de compression (1) qui est appliqué doucement à sa surface en soulevant le contrepoids (7). Un poids (12) est déposé sur le plateau de compression, puis retiré après un temps déterminé par le système automatique (B) et ce cycle est répété plusieurs fois. L'enregistreur trace la courbe des déformations de l'échantillon en fonction du temps.

Le poids utilisé et la fréquence du cycle de compression-recouvrance sont fonction de la nature de l'échantillon.

Dans le cas des fromages étudiés au paragraphe suivant, le poids était de $50 \mathrm{~g}$, les temps de compression et de recouvrance de $2 \mathrm{mn}$, $1 \mathrm{~mm}$ de déformation correspondait à $36 \mathrm{~mm}$ sur le graphique dont la vitesse de défilement était de $6 \mathrm{~mm}$ par minute.

\section{RESULTATS OBTENUS AVEC L'APPAREIL}

Cet appareil étant destiné à une étude sur l'affinage des fromages, il a été essayé sur le caillé-modèle mis au point pour ce travail $[8,10]$. La figure 2 représente le type de courbes obtenues sur ce type de fromage. L'échantillon soumis à une charge constante subit une déformation instantanée, suivie d'une déformation retardée qui se poursuit pendant un temps très long (fig. 2 a). Lorsque cesse la contrainte, à une recouvrance instantanée succède une recouvrance retardée qui est aussi très longue (fig. 2 b), mais l'échantillon ne recouvre pas sa hauteur initiale, il a subi une déformation permanente. Le fromage se comporte comme un corps visco-élastique ; des courbes de même allure ont été obtenues pour d'autres types de fromages, le Cheddar [5], l'Edam [12] ou le Gouda [7].

Pour des charges comprises entre 25 et $75 \mathrm{~g}$ les déformations de l'échantillon restent inférieures à $5 \mathrm{p} .100$ et sont pratiquement proportionnelles à la charge. D'autre part entre 25 et $100 \mathrm{~g}$, seules les courbes de complaisances obtenues pour des charges comprises entre 50 et $75 \mathrm{~g}$ sont pratiquement superposables, le fromage n'a donc un comportement visco-élastique linéaire que dans une zone 


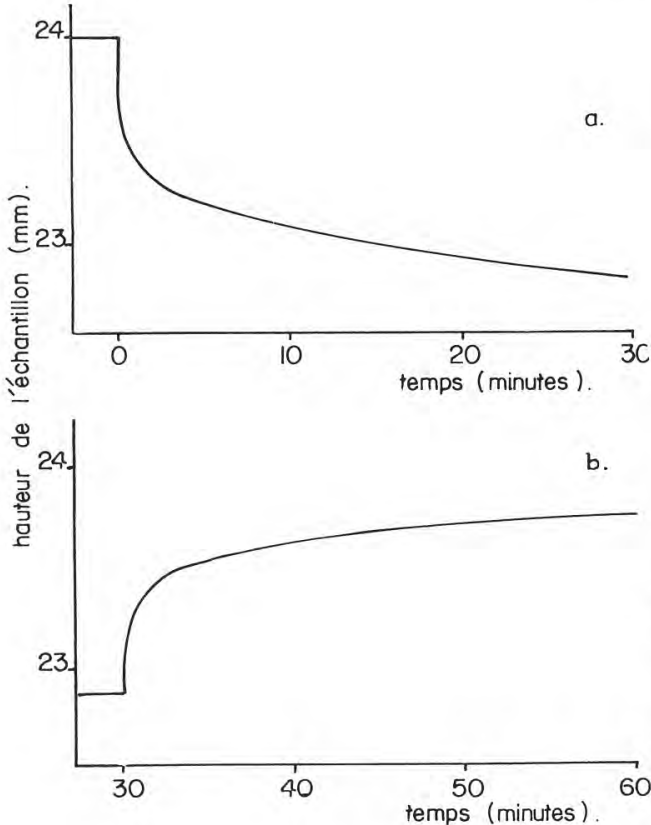

fig. 2

Courbes de compression (a) et de recouvrance (b) obtenues avec l'appareil.

Les deux courbes ont été enregistrées successivement sur le même échantillon provenant d'un fromage de $24 \mathrm{~h}$.

très restreinte. Finalement la charge de $50 \mathrm{~g}$ a été choisie pour tous nos essais.

Bien que la déformation et la recouvrance durent très longtemps (plus de $30 \mathrm{mn}$ ) (fig. 2 a et 2 b), nous avons choisi arbitrairement de limiter les durées de déformation et de recouvrance à $2 \mathrm{mn}$ car l'échantillon sèche rapidement et ses propriétés rhéologiques risqueraient d'être modifiées.

Si l'on soumet un échantillon à plusieurs cycles successifs de déformation et de recouvrance on constate que la première déformation est beaucoup plus importante que les suivantes qui sont semblables entre elles (fig. 3 et 4). D'après Davis [5] ceci vient de ce que le caillé n'est pas homogène et qu'il contient des gaz emprisonnés dans la masse et dans de petites ouvertures subsistant entre des grains de caillés mal soudés entre eux. Pour cette raison nous n'avons utilisé que les valeurs ( $C_{2}$ et $R_{2}$; fig. 3) obtenues au cours du deuxième cycle de compression-recouvrance pour calculer selon Davis [5] E et nฺ qui ont les dimensions d'un « module d'élasticité » et d'une « viscosité ».

(1) «Module d'élasticité »: $E=\frac{\mathrm{m} \cdot \mathrm{g} \cdot \mathrm{h} .}{3 \mathrm{AR}}$ 


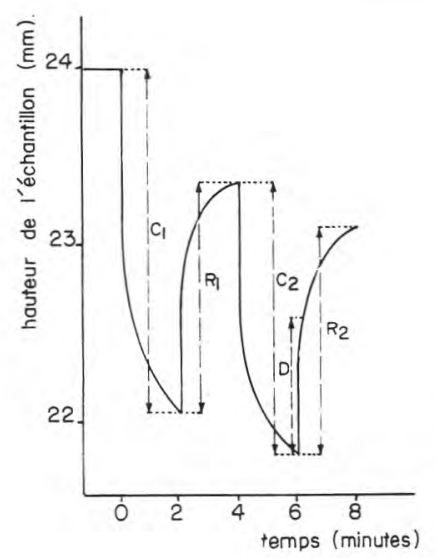

fig. 3

Courbes de déformation d'un échantillon do fromage soumis à deux compressions de $2 \mathrm{mn}$ séparées par une recouvrance de $2 \mathrm{mn}$.

(2) «Viscosité "

$$
: \eta=\frac{\text { m.g.h.t. }}{3 \mathrm{~A}(\mathrm{C}-\mathrm{R})}
$$

ou $\mathrm{m}=$ masse appliquée sur l'échantillon

$\mathrm{g}=$ accélération de la pesanteur

$\mathrm{h}=$ hauteur de l'échantillon

$\mathrm{A}=$ surface de l'échantillon

$\mathrm{t}=$ temps de compression

$\mathrm{C}=$ fluage

$\mathrm{R}=$ recouvrance

$\mathrm{C}-\mathrm{R}=$ déformation permanente

en unités S.I. ce «module d'élasticité » est exprimé en Pa et cette «viscosité » en Pa.s. (unité dénommée Poiseuille en France).

Il serait intéressant de connaître le « module d'élasticité instantanée » mais la recouvrance instantanée est difficile à mesurer avec précision dans nos conditions de mesure principalement à cause de la faible vitesse de déroulement du papier de l'enregistreur. Nous avons donc choisi de mesurer la valeur $\mathrm{D}$ qui est la recouvrance après $10 \mathrm{~s}$ (fig. 3), le temps le plus court mesurable sur l'enregistrement. Ce « module d'élasticité instantanée » peut être calculé en remplaçant $\mathrm{R}$ par $\mathrm{D}$ dans la formule [1].

L'appareil a été utilisé pour suivre l'évolution de la texture du caillé-modèle au cours d'une maturation de $46 \mathrm{j}$ à $12^{\circ} \mathrm{C}$. Les transformations biochimiques des constituants de ce caillé ne contenant que de la présure commerciale comme enzyme protéolytique ont été étudiées par ailleurs [8]. 


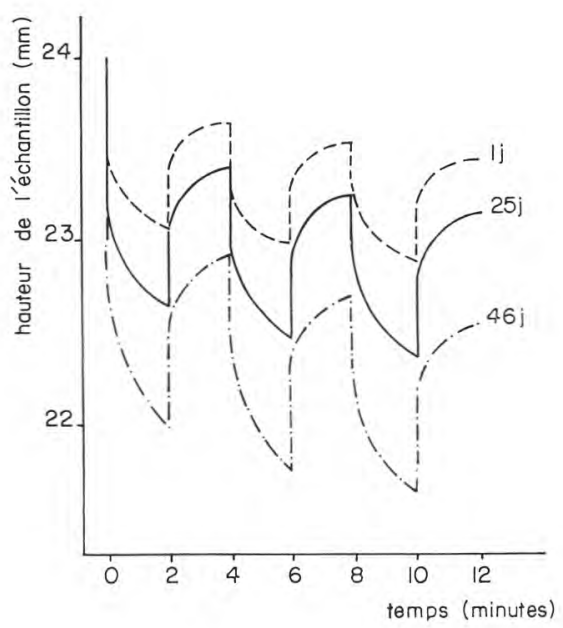

fig. 4

Exemple de courbes de déformation obtenues en fonction du temps d'affinage du fromage.

Les résultats obtenus en ce qui concerne sa texture sont donnés à titre d'exemple. La figure 4 montre que les courbes enregistrées à partir de ces fromages après $24 \mathrm{~h}, 25$ et $46 \mathrm{j}$ ont la même allure mais présentent des différences importantes quant à l'amplitude des déformations de l'échantillon. Ces fromages étant conditionnés sous paraffine, leur extrait sec est resté constant tout au long de l'affinage. Ce facteur n'intervient donc pas dans les différences observées.

Le tableau 1 montre des variations du " module d'élasticité » et de la " viscosité ". Ces deux paramètres diminuent progressivement au cours de la maturation du caillé et de manière significative. Rappelons que pour une viscosité donnée plus la valeur du " module d'élasticité » est faible et plus le fromage est déformable élastiquement et pour une élasticité donnée plus la viscosité est élevée et plus le fromage est ferme [5].

\section{CONCLUSION}

L'appareil qui a été mis au point est relativement simple, de réalisation peu coûteuse et d'un emploi facile. La façon de l'utiliser est d'ailleurs perfectible ne serait-ce qu'en utilisant un enregistreur plus rapide qui permettrait de définir avec plus de précision la déformation instantanée.

Sous sa forme actuelle l'appareil convient bien pour les études sur la maturation des fromages que nous avons entreprises $[8,16]$. 
TABLEAU 1. - Evolution de la texture du fromage au cours de la maturation

\begin{tabular}{|c|c|c|c|c|c|c|c|}
\hline $\begin{array}{l}\text { Nombre de } \\
\text { jours } \\
\text { d'affinage }\end{array}$ & $\begin{array}{c}\text { Fluage } \\
\text { C } \\
\left(\mathrm{m} \times 10^{-3}\right)\end{array}$ & $\begin{array}{c}\text { Recouvrance } \\
\mathrm{R} \\
\left(\mathrm{m} \times 10^{-3}\right)\end{array}$ & $\begin{array}{c}\text { Déformation } \\
\text { permanente } \\
\text { C-R } \\
\left(\mathrm{m} \times 10^{-3}\right)\end{array}$ & $\begin{array}{l}\text { Recouvrance } \\
\text { en } 10 \mathrm{~s} \mathrm{D} \\
\left(\mathrm{m} \times 10^{-3}\right)\end{array}$ & $\begin{array}{c}\text { "Viscosité " } \\
(\mathrm{n} \\
\left(\mathrm{Pa} . \mathrm{x} \times 10^{6}\right)\end{array}$ & $\begin{array}{l}\text { "Module } \\
\text { d'élasticité " E } \\
\left(\mathrm{Pa} \times 10^{3}\right)\end{array}$ & $\begin{array}{c}\text { "Module } \\
\text { d'élasticité } \\
\text { instantanée » } \\
\mathrm{E}_{\mathrm{i}}\left(\mathrm{Pa} \times 10^{3}\right)\end{array}$ \\
\hline 1 & 0,658 & 0,569 & 0,089 & 0,417 & 11,7 & 15,3 & 20,7 \\
\hline 11 & 0,644 & 0,578 & 0,066 & 0,417 & 15,8 & 15,0 & 20,7 \\
\hline 18 & 0,736 & 0,653 & 0,083 & 0,500 & 12,5 & 13,3 & 17,4 \\
\hline 25 & 0,888 & 0,772 & 0,116 & 0,583 & 9,0 & 11,2 & 15,0 \\
\hline 39 & 1,125 & 0,953 & 0,172 & 0,694 & 6,1 & 9,1 & 12,6 \\
\hline 46 & 1,111 & 0,919 & 0,192 & 0,639 & 5,4 & 9,4 & 13,6 \\
\hline
\end{tabular}


Il est en effet suffisamment sensible pour qu'on puisse constater des différences en cours d'affinage dans le cas le plus simple où la seule enzyme protéolytique est la présure comme le montrent les résultats ci-dessus. A fortiori, on peut donc penser qu'il permettra de mettre en évidence l'action d'autres enzymes, associées, seules ou en combinaison avec la présure ou de comparer entre eux des fromages fabriqués avec des enzymes différentes.

Cet appareil devrait également pouvoir être utilisé pour d'autres fromages comme le " rhéomètre » dont il est dérivé [5], soit pour suivre ou comparer leur état de maturation, soit à d'autres fins, par exemple pour comparer la texture à $24 \mathrm{~h}$ de fromages fabriqués selon des technologies différentes. Suivant la nature du fromage, il serait évidemment nécessaire d'adapter la méthode de mesure : durée de la compression, poids utilisé, etc.

Enfin, il pourrait être également utilisé pour d'autres produits alimentaires solides pour des essais de fluage mais aussi de pénétrométrie ou d'extrusion de gels.

\section{Remerciements}

Nous remercions vivement l'atelier de la Laiterie Expérimentale et plus spécialement $M$. Cavaroc pour la réalisation de l'appareil, et M. Launay (E.N.S.I. A.A., Massy) pour ses suggestions et critiques au cours de la rédaction du manuscrit.

\section{Rés u m é}

Le présent mémoire décrit un appareil simple qui a été mis au point pour apprécier la texture du fromage. Il permet de mesurer la déformation provoquée par une contrainte constante ainsi que la recouvrance élastique sur un échantillon découpé dans le fromage.

Cet appareil a été essayé sur un caillé modèle utilisé pour des études sur l'affinage des fromages. Les résultats montrent qu'il est suffisamment sensible pour détecter des différences de texture en cours d'affinage dans le cas le plus simple où la seule enzyme protéolytiaue est la présure.

\section{S u $\mathrm{m}$ m a ry}

A simple apparatus built to evaluate the cheese texture is described. It allows measurements of deformation produced by the application of a constant stress and the elastic recovery on a sample of cheese cut off.

This instrument was tested with a model-curd used in cheese ripening studies. Results show that it sensitivity is sufficient to 
detect differences during ripening when rennet is present as the only proteolytic enzyme.

Reçu pour publication en avril 1976.

\section{Références}

[1] Baron (M.) (1952). - The mechanical properties of cheese and butter. United Trade Press, London.

[2] BoHac (V.) (1966). - Uber das Messen von physikalischen Werten bei der Schmelzkäseezeugung. 17e Congr. Int. Lait., D, 263-266.

[3] Brennan (J. G.), Jowitt (R.) and Mughsi (O. A.) (1970), - Some experiences with the general food texturometer. J. Text. Stud., 1, 167-184.

[4] CAFFYN (J.C.) (1945). - The measurement of some physical properties of curd and cheese during manufacture of cheese. Dairy Ind., 10, 257-265.

[5] Davis (J. G.) (1937). - The rheology of cheese, butter and other milk products. J. Dairy Res., 8, 245-264.

[6] Emmon (D. B.) and Price (W. V.) (1959). - A curd firmness test for cottage cheese. J. Dairy Sci., 42, 553-556.

[7] Fukushima (M.), Taneya (S.) and Sone (T.) (1966). - Effect of emulsifying method and setting on viscoelasticity of processed cheese. J. Soc. Mat. Sci. Jap., 15, 287-290.

[8] Gripon (J. C.), Desmazeaud (M. J.), Le Bars (D.) et Bergetre (J. L.) (1975). Etude du rôle des micro-organismes et des enzymes au cours de la maturation des fromages. II. Influence de la présure commerciale. Le Lait, 55, 502-516.

[9] Kramer (A.) and SzczesniaK (A.S.) (1973), - Texture measurements of foods. Reidel Publishing Co., Boston (U.S.A.).

[10] Le Bars (D.), Desmazeaud (M. J.), Gripon (J. C.) et Bergère (J. L.) (1975). Etude du rôle des micro-organismes et de leurs enzymes dans la maturation des fromages. I. Fabrication d'un caillé modèle. Le Lait, 55, 377-389.

[11] MirgorodsKy (B.) (1966). - Method for determination of the cohesion of cheese grains in monolith. $17^{\mathrm{e}}$ Congr. Int. Lait, D, 421-424.

[12] MULdeR (H.) (1945). - Het bepalen van rheologische eigenschappen van kaas. Versl. Land. Onderzock, 51, 467-487.

[13] RaAdsweld (C. W.) and Mulier (H.) (1949). - The influence of the temperature on the ripening of Edam cheese. The Neth. Milk and Dairy J., 3, 117-141.

[14] Rogers (L. A.) and SANDERS (G.P.) (1942). - Devices for measuring physical properties of cheese. J. Dairy Sci., 25, 203-210.

[15] Scott-Blatr (G. W.) and Coppen (F.M. V.) (1941). - The consistency of cheese curd at the pitchingpoint and it's bearing on the firmness and quality of the finished cheese. J. Dairy Sci., 12, 44-54.

[16] Shf.ma (F.) and Sherman (P.) (1973). - Evaluation of some textural properties of foods with Instron Universal Testing Machine. J. Text. Stud., 4, 344-353.

[17] Sherman (P.) (1970). - Industrial Rheology, Academic Press, London.

[18] Szabo (G.) (1966). - Uber die rheologischen Eigenshaften des Schmelzkäses. 17e Congr. Int. Lait., D, 257-262.

[19] Templeton (H. L.) and Sommer (H. H.) (1930). - Some observation on processed cheese. J. Dairy Sci., 13, 203-219.

[20] Thomas (M. A.), Brown (F. B.) and Hanly (A. T.) (1970). - Studies in processed cheese manufacture : Instruments to measure firmness and elasticity in processed cheese. Aust. J. Dairy Techn., 25, 46-51. 